\title{
Cosmogenic Neutron Production at Daya Bay
}

\section{Xingtao Huang*}

Center for Particle Science and Technology, Shandong University

On behalf of Daya Bay collaboration

E-mail: huangxtdsdu.edu.cn

Spallation neutron from cosmic-ray muon is an important background for underground experiments, such as neutrino oscillation study, dark matter search, and neutrinoless double beta decay search. The neutron yield measurement results at Daya Bay were $Y_{n}=(10.26 \pm 0.86) \times 10^{-5}$, $(10.22 \pm 0.87) \times 10^{-5}$, and $(17.03 \pm 1.22) \times 10^{-5} \mu^{-1} \mathrm{~g}^{-1} \mathrm{~cm}^{2}$ at depths of 250,265 , and 860 meters-water-equivalent of the three experimental halls, respectively. The yield results were compared with other experiments as well as the simulated yield in FLUKA and GEANT4. A power law coefficient of $0.77 \pm 0.03$ was obtained by fitting the dependence of the neutron yield on muon energy [四].

The 39th International Conference on High Energy Physics (ICHEP2018)

4-11 July, 2018

Seoul, Korea

${ }^{*}$ Speaker. 
Spallation neutron from cosmic muon is a significant background for underground low-background experiments. Daya Bay Experiment consists of eight identically designed antineutrino detectors (ADs) filled with the Gd-doped liquid scintillator (GdLS) and muon detectors including the resistive plate chambers (RPCs), inner water shield (IWS) and outer water shield (OWS).

The neutron yield $Y_{n}$ is defined by $Y_{n}=\frac{N_{n}}{N_{\mu} L_{\text {avg }} \rho}$. Here $N_{\mu}$ is the number of muons passing through the GdLS region, $N_{n}$ is the number of neutrons produced in association with $N_{\mu}$ muons, $L_{\text {avg }}$ is the average path length of muons in the GdLS from simulation, and $\rho$ is the density of Daya Bay's GdLS. Muons are detected by the water pool and AD, accordingly they are tagged as the WS-muon and AD-muon $\left(N_{\mu, \text { Obs }}\right)$, respectively. $N_{\mu}$ is calculated from $N_{\mu}=N_{\mu, \text { Obs }} P_{\mu}, P_{\mu}$ is a correction factor and can be obtained from simulations.

In simulations, the sea level muon flux is described by a modified Gaisser formula, and the muons generated with MUSIC serve as the incident muon sample. The detector MC simulation is based on GEANT4, but neutrons are also simulated with FLUKA for the cross-check of GEANT4.

The analysis is based on 404 days of data collected with eight ADs and 217 days of data collected with six ADs. After corrections between the produced and detected number of neutrons with MC simulations, the neutron yield are measured as $Y_{n}=(10.26 \pm 0.86) \times 10^{-5},(10.22 \pm$ $0.87) \times 10^{-5}$, and $(17.03 \pm 1.22) \times 10^{-5} \mu^{-1} \mathrm{~g}^{-1} \mathrm{~cm}^{2}$ at depths of 250,265 , and 860 meters-waterequivalent of the three experimental halls, respectively.

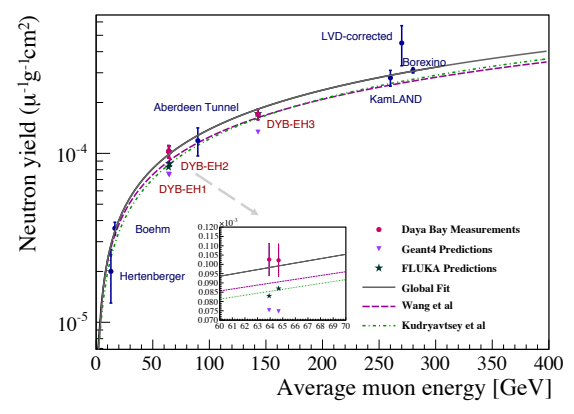

Figure 1: Neutron yield vs. average muon energy.

Comparisons of neutron yield measurements between the measured values of Daya Bay, the values from other experiments as well as the predicted values at Daya Bay from GEANT4 and FLUKA are performed. Previous studies have shown that the yield as a function of average muon energy can be described by a power-law $Y_{n}=a E_{\mu}^{b}$. Since Daya Bay has the unique capability to measure neutron production at three different underground experimental halls with essentially identical detectors, a power-law fit is applied to the measures in Fig.1 including the three data points from Daya Bay and yields coefficients $a=(4.0 \pm 0.6) \times 10^{-6} \mu^{-1} \mathrm{~g}^{-1} \mathrm{~cm}^{2}$ and $b=0.77 \pm 0.03$.

We acknowledge the supports from the National Natural Science Foundation of China (NSFC) under Contracts No.11175107 and the Joint Large-Scale Scientific Facility Funds of the NSFC and CAS under Contracts No. U1532258.

\section{References}

[1] Fengpeng An et al.(The Daya Bay Collaboration), Phys. Rev. D 97, 052009 (2018) 